\title{
Diagnóstico Energético em um Sistema de Abastecimento de Água
}

Energy Diagnosis in a Water Supply System

\author{
Carlos Frederico Dias Diniz ${ }^{1}$ \\ Luis Henrique Pereira da Silva ${ }^{1}$ \\ Sebastião Soares Lyra Netto ${ }^{1}$ \\ ${ }^{1}$ Escola Politécnica de Pernambuco, Universidade de Pernambuco, Recife, Brasil, \\ E-mail do autor principal: Carlos Frederico Dias Diniz carlosfd@poli.br
}

\section{Resumo}

Este trabalho objetiva desenvolver uma metodologia para diagnosticar energeticamente um sistema de bombeamento de água com vistas ao abastecimento de uma cidade. O referido método foi testado e validado para uma estação de bombeamento de água responsável pelo abastecimento de cerca de 40.000 pessoas. Dentre as ações propostas, chamam a atenção para a redução dos custos com energia elétrica a utilização de inversor de frequência, o redimensionamento de conjuntos motorbomba (CMBs), o enquadramento e a otimização tarifária. No caso testado, observou-se que a metodologia utilizada possui custo relativamente baixo e é de fácil aplicabilidade, podendo atingir um potencial de aproximadamente $27 \%$ de economia de energia elétrica, com tempo de retorno de investimento inferior a dois anos.

Palavras-Chave: Bombeamento de água; Diagnóstico energético; Eficiência energética; Economia de energia.

\begin{abstract}
This work intends to develop a methodology to do the energetic diagnose of a water pumping system in order to supply the population of a city. This method has been tested and validated for a pumping station of water responsible for supplying about 40,000 people. Among the proposed actions, those that draw attention to the reduction of electricity costs are the use of frequency inverter, set pumps (CMBs), framework and tariff optimization. In the tested situation, it was observed that the methodology used is simple, has a relatively low cost and is easy to apply, presenting a potential of about $27 \%$ of electricity savings, with a payback time of less than two years.
\end{abstract}

Key-words: Water pumping; Energy diagnosis; Energy efficiency; Energy saving. 


\section{Introdução}

Os sistemas de abastecimento de água e esgotamento sanitário são responsáveis por cerca de $3 \%$ da energia consumida no mundo. No Brasil, a situação não é diferente. "De acordo com dados do Programa Nacional de Conservação de Energia para o setor de Saneamento (Procel Sanear), obtidos em 2008, entre 2 e $3 \%$ do consumo total de energia elétrica no nosso país, o equivalente a cerca de 10 bilhões de kWh/ano, são consumidos por prestadoras de serviços de água e esgotamento sanitário" [1]. Aproximadamente, $90 \%$ dos gastos com energia elétrica das concessionárias de água devem-se às elevatórias dos sistemas de abastecimento público [2].

Um diagnóstico energético em um sistema de abastecimento pode indicar que simples ações administrativas e/ou operacionais podem implicar reduções de consumos e custos com energia elétrica, produzindo eficiência energética. Tais reduções podem ajudar tanto as empresas de saneamento, que podem ter sistemas mais eficientes e lucros maiores, quanto a população, que pode ter redução no valor das tarifas de energia elétrica a serem pagas. De acordo com Haddad e Martins [3], a eficiência energética pode ser definida como a capacidade de realizar um serviço e/ou produzir um bem com uma quantidade de energia inferior à que era usualmente consumida, sem que isso prejudique sua qualidade, conforto e eficiência.

A unidade escolhida para esse diagnóstico foi uma Estação Elevatória de Água Tratada (EEAT) do tipo Booster. Esta unidade pertence à Companhia Pernambucana de Saneamento (Compesa), está localizada no bairro de Prazeres, Jaboatão dos Guararapes, e é responsável pelo abastecimento de água de cerca de 40 mil pessoas.

\section{Objetivo}

Realizar um diagnóstico na busca de soluções para a eficiência energética e otimização de custos com o insumo energia elétrica em um sistema de abastecimento de água.

\section{Metodologia}

Para atingir os objetivos da pesquisa, o trabalho empregou os seguintes métodos:

- Análise das faturas de energia elétrica da unidade estudada;
- Realização de simulações tarifárias e análise dos resultados obtidos;

- Medição dos seguintes parâmetros elétricos: potências ativas $(\mathrm{kW})$, reativas (kvar), bem como o fator de potência (FP/cos);

- Avaliação do regime operacional da unidade;

- Análise termográfica dos motores de indução em funcionamento.

- Foram utilizados os seguintes equipamentos: um analisador de energia IMS Modelo P 600 e um termógrafo FLUKE TI -9.

\section{Resultados e Discussões}

Em visita às instalações da EEAT-Booster Prazeres, verificou-se que a alimentação elétrica é feita em média tensão $(13,8 \mathrm{kV})$, através de uma subestação aérea com um transformador de 225 kVA (Figura 1). Para a correção do fator de potência, há um banco capacitivo de 80 kvar, composto por quatro células de 20 kvar (Figura 2).

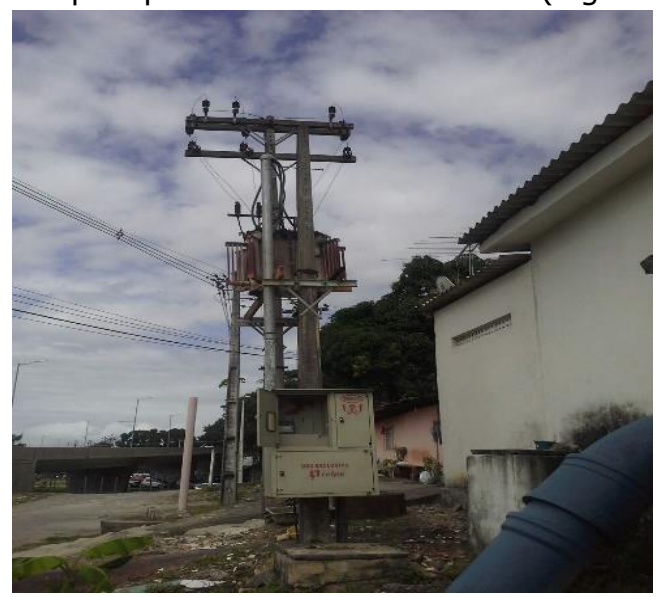

Figura 1: Subestação (SE) de 225 kVA. Fonte: os autores.

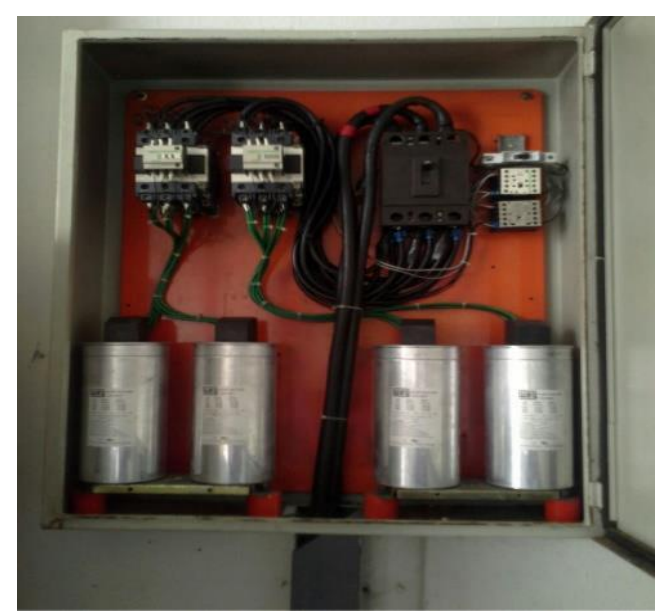

Figura 2: Banco capacitivo de 80 kvar. Fonte: os autores. 
A referida unidade possui dois conjuntos motorbomba (CMBs) de seis polos e $200 \mathrm{cv}$ de potência (Figura 3). São motores antigos (não foi possível identificar na placa o ano de fabricação), que já foram rebobinados algumas vezes e ainda utilizam chaves compensadoras na partida. Por uma estratégia operacional da Compesa, no momento do estudo, esta unidade estava voltando a operar depois de vários meses sem funcionar (Figuras $4 \mathrm{e}$ 5). A demanda contratada era de $30 \mathrm{~kW}$ na modalidade tarifária Alta Tensão Convencional, subgrupo A4 (de $2,3 \mathrm{kV}$ a $25 \mathrm{kV}$ ).

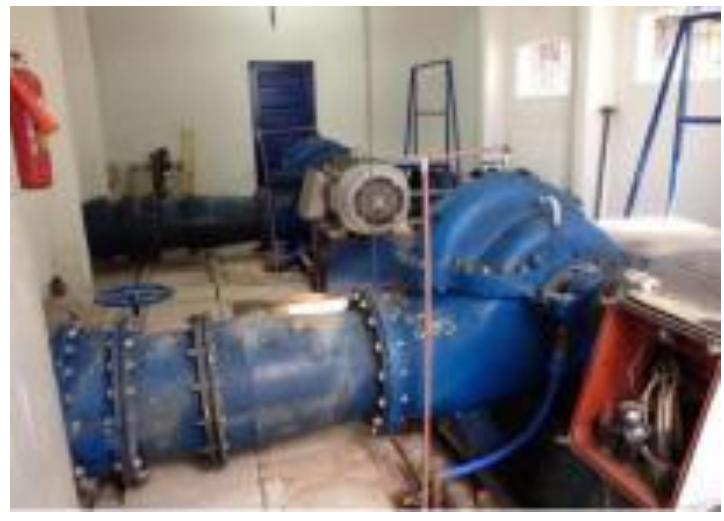

Figura 3: Conjuntos Motor-bomba. Fonte: os autores.

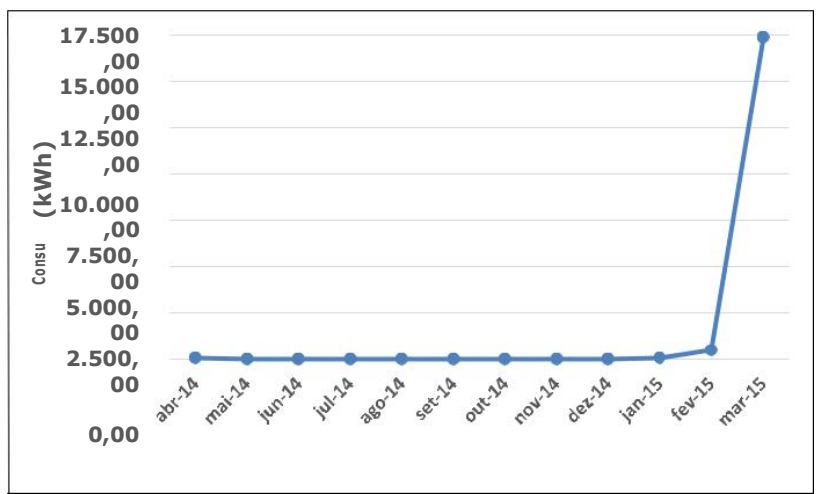

mês/ano

Figura 4: Histórico de consumo de energia elétrica. Fonte: os autores

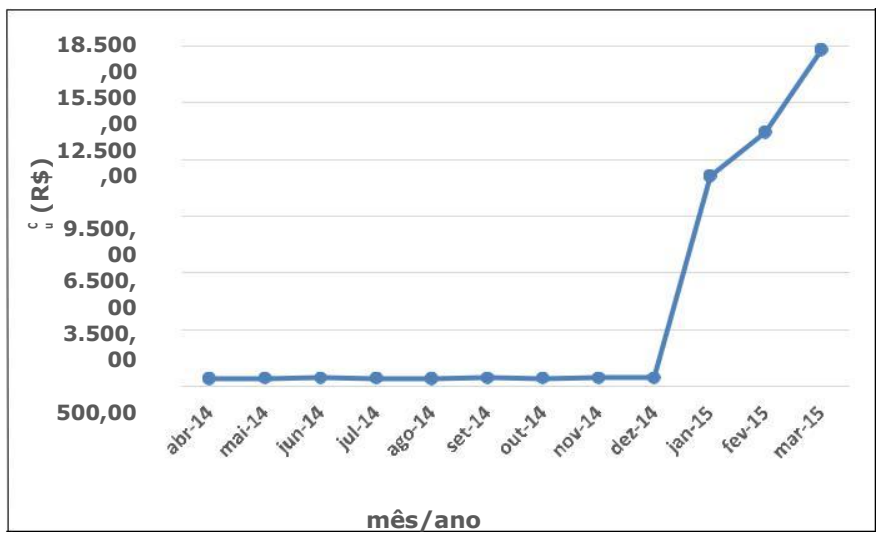

Figura 5: Histórico de custo com energia elétrica. Fonte: os autores.

O regime operacional proposto para o momento foi o de funcionamento de apenas um CMB, sendo o outro reserva, com parada no horário de ponta. Como não houve a solicitação à concessionária de energia do aumento da demanda contratada, observou-se a cobrança de multa por ultrapassagens da demanda contratada no valor médio mensal de $\mathrm{R} \$ 8.389,70$ nas primeiras faturas de energia pós-retorno da operação da unidade.

Considerando-se o Quadro 1 de tarifas de energia praticadas pela Companhia Energética de Pernambuco (Celpe) para o mês de agosto/2016, os consumos de energia elétrica médios mensais no horário fora de ponta e de ponta nos valores de $70.862 \mathrm{kWh}$ e $12.144 \mathrm{kWh}$, respectivamente, e as demandas a serem contratadas no horário fora de ponta e de ponta nos valores de $145 \mathrm{~kW}$ e $30 \mathrm{~kW}$, respectivamente, foi possível simular os valores das faturas, conforme valores obtidos no Quadro 2.

Quadro 1: Tarifas de energia elétrica - agosto/2016.

\begin{tabular}{|c|c|c|c|c|}
\hline & \multicolumn{2}{|c|}{ Consumo (R\$/kWh) } & \multicolumn{2}{|c|}{ Demanda (RST/kW) } \\
\hline & Ponta & Fora Ponta & Ponta & Fora Ponta \\
\hline THV (A4) & 1,48948 & 0,31801 & \multicolumn{2}{|c|}{14,38828} \\
\hline THA (A4) & 0,47063 & 0,31801 & 41,99478 & 14,38828 \\
\hline ATC (A4) & \multicolumn{2}{|c|}{0,34917} & \multicolumn{2}{|c|}{47,11248} \\
\hline
\end{tabular}

Fonte: Celpe, 2016.

Legendas:

THV - Tarifa Horária-Verde;

THA - Tarifa Horária-Azul;

TATC - Tarifa Alta Tensão Convencional. 
Quadro 2: Simulações tarifárias. EAT-BOOSTER PRAZERES - 1 CMB DE 200 cV COM PARADA APENAS NO HORÁROO DE PONTA \begin{tabular}{|c|c|c|c|c|c|c|c|}
\hline Unidade & $\begin{array}{c}\text { Cons. Pla } \\
(\mathrm{kWh})\end{array}$ & $\begin{array}{c}\text { Cons. FPla } \\
(\mathrm{kWh})\end{array}$ & $\begin{array}{c}\text { Dem } \\
\text { Pla }(\mathrm{kW})\end{array}$ & $\begin{array}{c}\text { Dem } \\
\text { Fra }(\mathrm{kW})\end{array}$ & TATC(RS) & THN(PS) & THA(RS) \\
\hline BOOSTRR PRAZERES & $12.144,00$ & $70.862,00$ & 30 & 145 & $35.814,51$ & $42.709,37$ & $31.596,30$ \\
\hline
\end{tabular}

Fonte: os autores.

Onde:

Cons. Pta. - Consumo médio no horário de ponta; Cons. FPta. - Consumo médio no horário fora de ponta;

Dem. Pta. - Demanda contratada no horário de ponta;

Dem. FPta. - Demanda contratada no horário fora de ponta.

Observa-se que uma simples modificação de modalidade tarifária de TATC para THA traria uma economia mensal na fatura de energia estimada em $\mathrm{R} \$ 4.218,21$.

No Quadro 3, temos os valores obtidos, em intervalos de 15 minutos, através do instrumento IMS para as potências ativa, reativa e o fator de potência (FP).

Quadro 3: Parâmetros elétricos medidos.

\begin{tabular}{|c|c|c|c|c|c|c|c|c|c|c|}
\cline { 2 - 10 } \multicolumn{1}{c|}{} & 09h15 & $09 h 30$ & 09 h5 & 10 h00 & 10 h15 & 10 h30 & 10 h45 & 11 h00 & 11 h15 & 11 h30 \\
\hline P. Ativa (kW) & 101,36 & 135,84 & 129,74 & 126,64 & 126,32 & 126,04 & 126,02 & 126,08 & 125,84 & 125,42 \\
\hline P. Reativa (kvar) & 83,52 & 107,9 & 106,5 & 105,74 & 106,1 & 106,28 & 106,46 & 106,4 & 106,46 & 106,18 \\
\hline FP & 0,77 & 0,78 & 0,77 & 0,77 & 0,77 & 0.76 & 0,76 & 0,76 & 0.76 & 0,76 \\
\hline
\end{tabular}

Fonte: os autores.

A partir deste experimento, foi observado que o banco capacitivo não estava funcionando corretamente de forma que a média do FP foi de 0,77 , e não 0,98 , conforme esperado pela potência reativa instalada (80 kvar). Uma correção deste mau funcionamento traria uma economia mensal na fatura de energia estimada em $\mathrm{R} \$ 949,16$, sendo esse valor o médio cobrado pelos reativos excedentes.

O controle de vazão da unidade estudada é feito através de estrangulamento de uma válvula manual, ou seja, o operador está alterando a curva do sistema para controlar a vazão. Quando estrangulamos uma válvula, na partida ou no controle de vazão de uma bomba, nós alteramos a curva do sistema (Figura 6) [4].

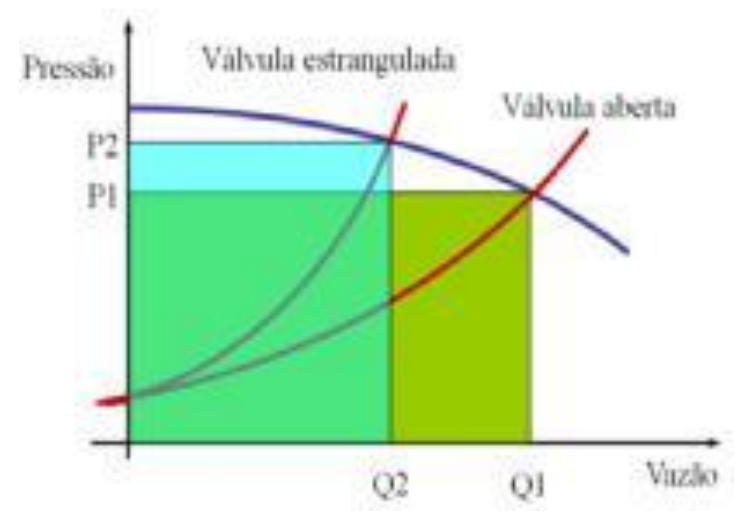

Figura 6: Controle de vazão através da manobra de válvula.

Fonte: MADDARENA, 2006

Atualmente, o controle eletrônico da velocidade dos motores é mais eficaz que o estrangulamento de válvulas e permite uma interessante economia de energia. Observa-se que a curva da bomba é alterada, e não a do sistema (Figura 7) [4].

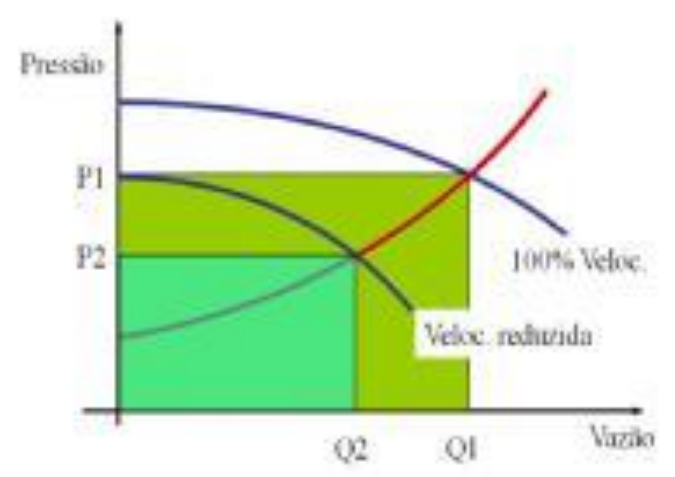

Figura 7: Controle de vazão através da variação da rotação da bomba.

Fonte: MADDARENA, 2006.

Como a potência elétrica é proporcional à área do retângulo delimitado pelo ponto de operação e à origem dos eixos, nota-se facilmente que, reduzindo a velocidade da bomba para diminuir a vazão, teremos uma economia de energia maior e mais eficiente que o método do estrangulamento de válvulas.

Para o controle de vazão de um CMB, a utilização de um inversor de frequência nos dá uma economia de energia bem maior que o método do estrangulamento. De acordo com a curva de ensaio, uma redução de $10 \%$ na velocidade do motor, o que acarretaria uma redução de $10 \%$ na vazão da bomba, daria uma diminuição de mais de $20 \%$ da potência elétrica solicitada pelo motor. 
Para facilitar a visualização, apresentamos o gráfico exposto na Figura 8.

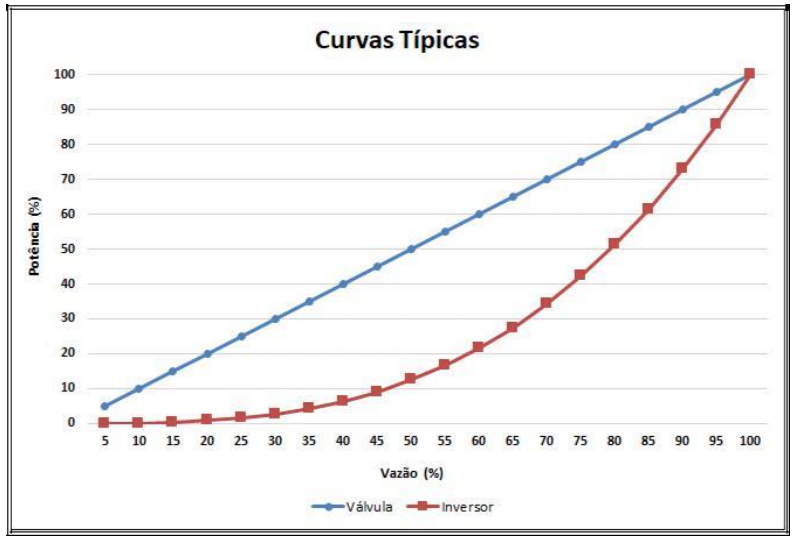

Figura 8: Comparação através de ensaio de controle de vazão entre o método de válvula de controle (estrangulamento) e um acionamento eletrônico (inversor de frequência) Fonte: os autores

O inversor de frequência é um equipamento eletrônico capaz de produzir uma variação nos valores de frequência da tensão elétrica que alimenta o motor, causando uma variação na sua velocidade de sua rotação [5].

Em uma bomba com motor de $200 \mathrm{cv}(147,2$ $\mathrm{kW})$ com rotação nominal de $1.200 \mathrm{rpm}$, diminuindo em $10 \%$ sua rotação, tem-se:

$\mathrm{N} 1=1.200 \mathrm{rpm}$

$\mathrm{N} 2=1.080 \mathrm{rpm}$

$\mathrm{P} 1=200 \mathrm{cv}=147,2 \mathrm{~kW}$ (Potência nominal com o motor a rotação plena).

Relacionamos as potências com as velocidades da seguinte forma:

$$
\begin{aligned}
& (\mathrm{P} 2 / \mathrm{P} 1)=(\mathrm{N} 2 / \mathrm{N} 1)^{3} \\
& \mathrm{P} 2=(\mathrm{N} 2 / \mathrm{N} 1)^{3} \cdot \mathrm{P} 1=(1.080 / 1.200)^{3} \cdot 147,2 \\
& \mathrm{P} 2=107,31 \mathrm{~kW}
\end{aligned}
$$

O resultado acima nos mostra uma redução de $27,1 \%$ na potência elétrica solicitada pela carga. De acordo com os resultados das medições e das tarifas de energia, uma economia de $27,1 \%$ no consumo de energia representaria um montante de $R \$ 6.612,88$, significando uma redução de 20,93 $\%$ na fatura de energia elétrica quando comparamos os montantes "THA" presentes nos Quadros 2 e 4.
Quadro 4: Simulações tarifárias com a redução de potência.

\begin{tabular}{l}
\hline \multicolumn{10}{|c|}{ EAT-BOOSTER PRAZERES - 1 CMB DE 200 cV COM PARADA APENAS NO HORÁRIO DE PONTA } \\
\begin{tabular}{|c|c|c|c|c|c|c|c|}
\hline Unidade & $\begin{array}{c}\text { Cons. Pta } \\
(\mathrm{kWh})\end{array}$ & $\begin{array}{c}\text { Cons. FPta } \\
(\mathrm{kWh})\end{array}$ & $\begin{array}{c}\text { Dem. Pta } \\
(\mathrm{kW})\end{array}$ & $\begin{array}{c}\text { Dem. FPta } \\
(\mathrm{kW})\end{array}$ & TATC(RS) $)$ & THV(Rs) & THA(Rs) \\
\hline BOOSTER PRAZERES & $12.144,00$ & $51.651,00$ & 30 & 110 & $27.457,67$ & 36.096 .49 & 24.983 .42 \\
\hline
\end{tabular}
\end{tabular}

Fonte: os autores.

Os CMBs da unidade operam atualmente com motores antigos e que já foram rebobinados várias vezes. Rebobinamento em desacordo com os dados originais de projeto do fabricante: nesse caso, o número de espiras ou bitola de fio diferentes do original colocam a condição de funcionamento do motor fora das características ideais, acarretando decréscimo no rendimento e aumento nas perdas. Pesquisas mostram que, a cada rebobinamento, um motor perde em média $3 \%$ do seu rendimento [6].

Além disso, os termogramas apresentados nas Figuras 9 e 10 mostram altas temperaturas nos motores em regime operacional.

Observação: As temperaturas que aparecem nas imagens termográficas estão na escala Fahrenheit.

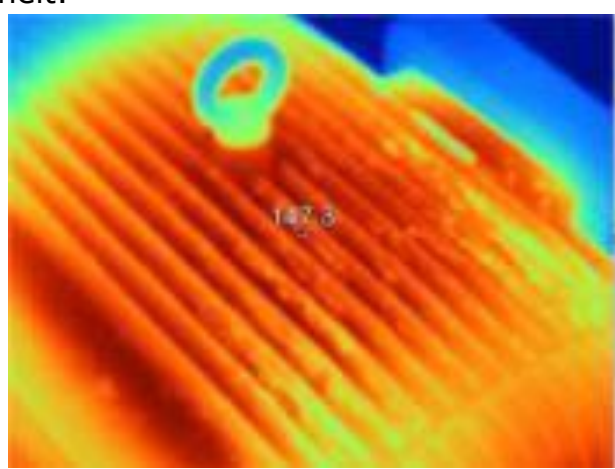

Figura 9: Vista superior do motor $-64,06^{\circ} \mathrm{C}$. Fonte: os autores.

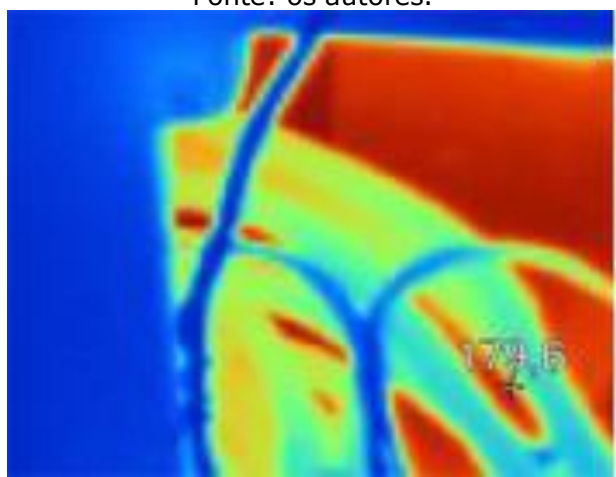

Figura 10: Condutores na caixa de ligação $-82^{\circ} \mathrm{C}$ Fonte: os autores.

Temperaturas elevadas podem afetar a vida e a confiabilidade dos motores, causando a 
deterioração de suas partes vitais, especialmente a isolação [6].

O Decreto $n^{\circ} 4.508$, de 11 de dezembro de 2002, que dispõe sobre a regulamentação específica sobre os níveis mínimos de eficiência energética de motores elétricos trifásicos de indução rotor gaiola de esquilo, especifica valores mínimos de rendimento para motores classificados como padrão (motores da linha standard ou convencional). Detalha também valores mínimos de rendimento para uma linha de motores denominados de alto rendimento [6].

A substituição dos motores da linha padrão por motores de alto rendimento (A.R.) poderá trazer uma economia mensal interessante na fatura de energia elétrica.

Considerando-se uma redução de $10 \mathrm{~kW}$ de potência solicitada da rede elétrica pela substituição dos motores da linha padrão da unidade estudada por motores de alto rendimento, estimamos uma economia mensal de $\mathrm{R} \$ 1.637,26$ na fatura de energia elétrica quando comparamos os montantes "THA" presentes nos Quadros 4 e 5.

Quadro 5: Simulações tarifárias com a utilização de motores de alto rendimento

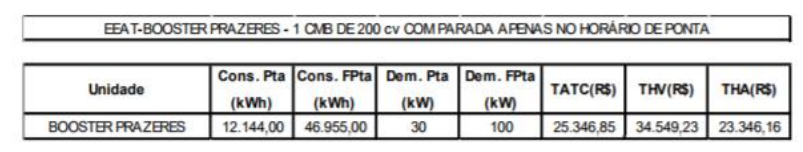

Fonte: os autores.

Na prática, as perdas totais do motor elétrico de alto rendimento correspondem a aproximadamente $60 \%$ das perdas totais de um motor elétrico comum. Contudo, apesar desta redução substancial das perdas, o rendimento do motor de alto rendimento permanece em torno de $3,5 \%$ maior que o rendimento do motor elétrico standard.

\section{Conclusão}

De acordo com os resultados das simulações, pode-se obter uma economia substancial com a manutenção do banco capacitivo, troca do sistema atual para o sistema com acionamento eletrônico, substituição dos motores obsoletos ou standard (padrão) por motores de alto rendimento e modificação do sistema tarifário para a modalidade horária-azul (com desligamento no horário de ponta). A demanda contratada com a concessionária de energia será reduzida de 145 6 para $100 \mathrm{~kW}$ no horário fora de ponta e $30 \mathrm{~kW}$ no horário de ponta em consequência das ações tomadas.

Quantificando os valores, teremos:

a) Economia mensal estimada com a manutenção do banco capacitivo da unidade: $R \$ 949,16$.

b) Economia mensal estimada com a mudança dos acionamentos dos CMBs e ajustes na modalidade tarifária e com a adequação do contrato de demanda: $\mathrm{R} \$ 6.612,88$.

c) Economia mensal estimada na fatura de energia pela da troca dos motores da linha padrão por motores de alto rendimento e readequação do contrato de demanda: $\mathrm{R} \$ 1.637,26$.

d) Investimento estimado na aquisição de dois motores de alto rendimento $200 \mathrm{cv}$, seis polos: $R \$ 60.000,00$.

e) Investimento estimado na aquisição de um inversor de frequência próprio para acionar esses motores: R\$40.000,00.

f) Investimento estimado na aquisição de um quadro com modelo de distribuição geral: $\mathrm{R} \$$ $60.000,00$.

Observação: Para acionar os CMBs basta adquirir apenas um inversor de frequência, uma vez que apenas um CMB funciona por vez.

$O$ investimento total estimado seria de $R \$$ $160.000,00$ (valores aproximados sem contabilizar os custos da mão de obra para as montagens) e a economia mensal estimada seria de $R \$ 9.199,30$.

Observa-se facilmente que, por payback simples, o investimento teria retorno simples em 17,39 meses (poderemos considerar 18 meses).

\section{Apoio Institucional}

A pesquisa resumida neste artigo contou com o apoio do Grupo de Gestão da Energia Elétrica da Escola Politécnica de Pernambuco (POLI/UPE), vinculado ao curso de Engenharia Elétrica Eletrotécnica desta mesma instituição.

\section{Referências}

[1] H. P. GOMES. Sistemas de Saneamento Efici-ência Energética. Editora Universitária, João Pessoa-PB, p.3, 2010. $1^{\text {a }}$ edição. 366p.

[2] M. T. TSUTIYA. Redução do custo de energia elétrica em sistemas de abastecimento de água. Associação Brasileira de Engenharia Sanitária e 
Ambiental, São Paulo-SP, 2005. $1^{\text {a }}$ edição. 376p.

[3] J. HADDAD, A. R. MARTINS. Conservação de Energia: eficiência energética de instalações e equipamentos. Editora da EFEI, Itajubá-MG, 2001. $1^{\text {a }}$ edição. 467p.

[4] E. MADDARENA. Aplicação de Inversores de Frequência em Sistemas de Bombeamento. Brasília-DF, p.14-18, 2006.

[5] M.T. TSUTIYA. Abastecimento de Água. Departmento de Engenharia Hidráulica e Sanitária - USP, São Paulo-SP, 2005. $2^{\text {a }}$ edição. $643 p$.

[6] PROCEL INDÚSTRIA-Edição Seriada/ELETROBRAS-CNI/IEL. Motor Elétrico-Guia Básico. Brasília-DF, 2009. 